\title{
The association between neutrophil-to-lymphocyte ratio and anaphylaxis refractory to epinephrine treatment
}

\author{
Kiwook Kim ${ }^{1}$, Kyoung Ho Choi ${ }^{1}$, , Jung Taek Park ${ }^{1}$, Joo Suk Oh${ }^{1}$, Doo Hyo Lee ${ }^{1}$, \\ Hyun Ho Jeong ${ }^{1}$, Young Min Oh${ }^{1}$, Se Min Choi ${ }^{1}$
}

\author{
${ }^{1}$ Department of Emergency Medicine, \\ Uijeongbu St. Mary's Hospital, \\ Uijeongbu-si, Republic of Korea \\ *Correspondence \\ ergogo@catholic.ac.kr \\ (Kyoung Ho Choi)
}

\begin{abstract}
Objectives: Anaphylaxis refractory to epinephrine treatment is a potentially fatal condition requiring additional medications. Neutrophil-to-lymphocyte ratio (NLR) is commonly used to predict severity in allergic diseases. The aim of this study was to determine the association between NLR and refractory anaphylaxis.

Methods: This was a retrospective, observational study of 126 adult anaphylaxis patients arriving at the emergency department between January 2015 and December 2019. Patients were placed into refractory anaphylaxis, if they required more than two 0.3 $\mathrm{mg}$ injections of intramuscular epinephrine for symptom resolution, and non-refractory anaphylaxis groups. NLRs were determined at the time of arrival at the hospital and were compared between groups.

Results: Thirty-two (25.4\%) patients were categorized as refractory anaphylaxis cases. NLR was significantly lower in the refractory anaphylaxis than in the non-refractory anaphylaxis group $(P<0.001)$. In the multivariate logistic regression analysis model, NLR was inversely associated with the occurrence of refractory anaphylaxis (adjusted odds ratio $0.33,95 \%$ confidence interval $0.13-0.81, P=0.016$ ). The area under the receiver operating characteristic curve of NLR for prediction of refractory anaphylaxis was $0.717(P<0.001)$. The optimal cut-off value of NLR was $<0.68$ using the Youden index, with $50.0 \%$ sensitivity and $80.9 \%$ specificity.

Conclusions: NLR was independently and inversely associated with the occurrence of refractory anaphylaxis among anaphylactic patients. Therefore, NLR has the potential to be used as an easy and inexpensive test to predict refractory anaphylaxis in patients.
\end{abstract}

\section{Keywords}

Anaphylaxis; Rrefractory anaphylaxis; Neutrophil-to-lymphocyte ratio; White blood cells

\section{Introduction}

Anaphylaxis is a serious, potentially fatal type I allergic reaction that involves multiple organ systems [1]. It is primarily mediated by mast cells and basophils, which induce systematic hypersensitivity reactions such as airway compromise and distributive shock by releasing inflammatory mediators [2]. The cornerstone of anaphylaxis treatment is epinephrine injection [3]. Epinephrine is a nonselective $\alpha$ - and $\beta$-adrenergic agonist, which counteracts anaphylactic reactions by reducing tissue edema, preventing hypotension, increasing heart rate and cardiac contractility, and dilating airways. Most patients respond well to proper disease management, limiting fatal cases to less than $0.3 \%$ of all anaphylaxis patients in the United States [4]. However, some patients follow a more severe clinical course, where symptoms are refractory to intramuscular epinephrine injections [5]. According to a previous study, $0.37 \%$ of all anaphylaxis cases required more than two intramuscular epinephrine injections [6]. These patients, in whom anaphylaxis was triggered mostly by drugs, were at a higher risk of death and required admission to the intensive care unit and additional treatment measures. Thus, it may be critical to promptly predict and recognize these epinephrine-refractory cases. However, currently, there are no tests available in predicting refractory anaphylaxis.

Several laboratory tests have been previously evaluated in predicting the severity of allergic reactions. The neutrophilto-lymphocyte ratio (NLR) is a simple serum marker, obtained by performing a complete blood count (CBC) with differential tests, which reflects the immune status of the subject. Since it is easily measured, reproducible, and inexpensive, previous studies have investigated the association between NLR and allergic diseases [7-9]. These studies have shown that a higher NLR was associated with a more severe reaction in allergic rhinitis, atopic dermatitis, and asthma. However, the association between NLR and the occurrence of refractory anaphylaxis has not been studied previously. 
TA B L E 1. Criteria for diagnosing anaphylaxis [10].

Anaphylaxis is highly likely when any one of the following three criteria are fulfilled, all within minutes to several hours:

1. Acute onset of an illness with involvement of the skin, mucosal tissue, or both (e.g., generalized hives, pruritis or flushing, swollen lips-tongue-uvula) and at least one of the following:

a. Respiratory compromise (e.g., dyspnea, wheeze-bronchospasm, stridor, reduced peak expiratory flow, hypoxemia)

b. Reduced blood pressure or associated symptoms of end-organ dysfunction (e.g., hypotonia or collapse, syncope, incontinence)

2. Two or more of the following that occur rapidly after exposure to a likely allergen for that patient:

a. Involvement of the skin-mucosal tissue

b. Respiratory compromise

c. Reduced blood pressure or associated symptoms

d. Persistent gastrointestinal symptoms (e.g., crampy abdominal pain, vomiting)

3. Reduced blood pressure after exposure to known allergen for that patient:

a. Adults: systolic blood pressure of less than $90 \mathrm{mmHg}$ or greater than $30 \%$ decrease from that person's baseline

b. Infants and children: greater than $30 \%$ decrease in systolic blood pressure from that person's baseline, or

* systolic blood pressure less than $70 \mathrm{mmHg}$ from 1 month to 1 year

* systolic blood pressure less than $70 \mathrm{mmHg}+(2 \mathrm{x}$ age $)$ from 1 to 10 years

* systolic blood pressure less than $90 \mathrm{mmHg}$ from 11 to 17 years

TA B L E 2. Grading system for generalized hypersensitivity reactions [11].

\begin{tabular}{|c|c|}
\hline Grade & Defined by \\
\hline 1 - Mild (skin and subcutaneous tissues only) & Generalized erythema, urticaria, periorbital edema, or angioedema \\
\hline $\begin{array}{l}2 \text { - Moderate (features suggesting respiratory, } \\
\text { cardiovascular, or gastrointestinal involvement) }\end{array}$ & $\begin{array}{l}\text { Dyspnea, stridor, wheeze, nausea, vomiting, dizziness (presyncope), } \\
\text { diaphoresis, chest or throat tightness, or abdominal pain }\end{array}$ \\
\hline $\begin{array}{l}3 \text { - Severe (hypoxia, hypotension, or neurologic } \\
\text { compromise) }\end{array}$ & $\begin{array}{l}\text { Cyanosis or oxygen saturation }<92 \% \text { at any stage, hypotension (systolic } \\
\text { blood pressure }<90 \mathrm{mmHg} \text { in adults), confusion, collapse, loss of } \\
\text { consciousness, or incontinence }\end{array}$ \\
\hline
\end{tabular}

In this study, our aim was to determine the association between NLR and the occurrence of anaphylaxis refractory to epinephrine treatment.

\section{Methods}

\subsection{Study design and population}

This was a retrospective, observational study that enrolled anaphylaxis patients arriving at a regional emergency department (ED) of a teaching hospital in South Korea between January 2015 and December 2019. Adults (> 18 years) who were diagnosed with moderate to severe anaphylaxis, according to the criteria of the National Institute of Allergy and Infectious Disease (NIAID) and the Food Allergy and Anaphylaxis Network (FAAN), and the grading system suggested by Brown SG were included in the study $[10,11]$. The diagnostic criteria and the grading systems for anaphylaxis are described in Table 1 and Table 2. Patients who were transferred in from other hospitals, who suffered prehospital cardiac arrest, or who had no laboratory data available were excluded.

The term 'refractory anaphylaxis' is not clearly defined. We used the definition from a previous study [6], which described refractory anaphylaxis as a condition that required more than two doses of $0.3 \mathrm{mg}$ intramuscular epinephrine for complete symptom resolution. According to the definition, the enrolled patients were divided into two groups: refractory and non- refractory anaphylaxis. Our study was approved by the Institutional Review Board of Uijeongbu St. Mary's Hospital on March 25, 2020 (number UC20RASI0037).

\subsection{Anaphylaxis management}

Patients with anaphylaxis were treated by following previously established guidelines [1]. Epinephrine $0.3 \mathrm{mg}(0.3 \mathrm{~mL}$ of a 1 $\mathrm{mg} / \mathrm{mL}(1: 1,000)$ solution) was injected via an intramuscular route as soon as anaphylaxis was recognized. A repeat injection of intramuscular epinephrine was ordered if symptoms did not resolve after five or more minutes after the first injection. If symptoms persisted, intravenous infusion of epinephrine or other vasopressors such as dopamine or norepinephrine were considered. Intubation or other resuscitative measures were taken as needed.

We recommended that all patients be admitted for prolonged observation. Patients with persisting symptoms, or who were being treated by an intravenous infusion of epinephrine, dopamine, or norepinephrine, or who were intubated were admitted to the intensive care unit for proper management.

\subsection{Data collection}

We collected data on patient demographics and characteristics via electronic medical records. Data collected were as follows: age, sex, use of emergency medical services, past history 
of diseases (hypertension, diabetes mellitus (DM), cardiovascular disease, chronic pulmonary disease, and malignancy), known previous allergies to elicitors, elicitors that caused anaphylaxis (categorized as food, drugs, contrast media, insect venom, others, and idiopathic), symptoms related to skin and mucous tissue (skin rash, pruritis, swelling of lips, tongue and uvula), cardiovascular symptoms (hypotension, dizziness, collapse, loss of consciousness, and incontinence), respiratory symptoms (dyspnea, wheezing, stridor, and hypoxemia), and gastrointestinal symptoms (cramping abdominal pain and intractable vomiting), initial mean arterial pressure (MAP), heart rate, body temperature, number of intramuscular epinephrine injections, dose of administered intravenous epinephrine (mg), use of vasopressors (dopamine or norepinephrine), $\mathrm{H}_{1}$ blocker, $\mathrm{H}_{2}$ blocker, and corticosteroids, rate of hospital admission, and hospital length of stay in days.

Initial laboratory findings were also collected at the time of ED arrival as follows: hemoglobin $(\mathrm{g} / \mathrm{dL})$, platelet count $\left(10^{9} / \mathrm{L}\right)$, white blood cell (WBC) count $\left(10^{9} / \mathrm{L}\right)$ with differentials, which included percentages (\%) and absolute count $\left(10^{9} / \mathrm{L}\right)$ of segmented neutrophils, lymphocytes, monocytes, eosinophils, and basophils, glucose $(\mathrm{mg} / \mathrm{dL})$, blood urea nitrogen $(\mathrm{mg} / \mathrm{dL})$, creatinine $(\mathrm{mg} / \mathrm{dL})$, sodium $(\mathrm{mmol} / \mathrm{L})$, potassium $(\mathrm{mmol} / \mathrm{L})$, chloride $(\mathrm{mmol} / \mathrm{L})$, and C-reactive protein $(\mathrm{mg} / \mathrm{L})$. CBC was measured using an automated blood cell counter (Sysmex XE-2100). NLR was calculated as the ratio of segmented neutrophil counts to lymphocyte counts in the same blood sample.

\subsection{Statistical analysis}

Categorical variables are displayed as numbers (percentages). All continuous variables were tested for normal distribution using the Shapiro-Wilk test and are presented as the means \pm standard deviation (SD) for normally distributed data and as medians (interquartile range (IQR)) for data not normally distributed. For comparison of patient characteristics and laboratory findings between groups, the chi-square test and Fisher's exact test for categorical variables, the Student's $t$ test for normally distributed continuous variables, and the Mann-Whitney test for non-normally distributed continuous variables were used. The independent association between baseline characteristics, including NLRs, and the occurrence of refractory anaphylaxis were analyzed using multivariate logistic regression. Variables with a $P$ value $<0.1$ on univariate logistic regression analysis were manually entered into the model. Furthermore, the receiver operating characteristic (ROC) curve was drawn and the area under the curve (AUC) was calculated to determine whether NLR was a good predictor of refractory anaphylaxis. The optimal cut-off value was obtained by calculating the Youden index.

All statistical analyses were performed using MedCalc Statistical Software version 19.6 (MedCalc Software, Ostend, Belgium) and GraphPad Prism version 9.0.0 (GraphPad Software, San Diego, California, USA). A $P$ value $<0.05$ was considered statistically significant.

\section{Results}

Fig. 1 shows the flow chart of the study design. Of the 164 anaphylaxis patients who were included in the study, a total of 38 patients were excluded because they were transferred in from other hospitals $(\mathrm{n}=32)$, suffered prehospital cardiac arrest $(n=3)$, or did not have laboratory data $(n=3)$. 126 patients were eventually enrolled. The mean age was $54.3 \pm$ 12.6 years, and $70(55.6 \%)$ patients were male. Drugs were the most common allergic elicitors $(n=52,41.3 \%)$, followed by insect venom $(n=29,23.0 \%)$, food $(n=24,19.0 \%)$, contrast media $(n=9,7.1 \%)$, and others, which included exercise and cigarette smoke $(n=2,1.6 \%) .10$ patients $(7.9 \%)$ had no definite trigger of anaphylaxis. Skin symptoms were most common $(\mathrm{n}=106,84.1 \%)$, followed by cardiovascular $(\mathrm{n}=$ $105,83.3 \%)$, respiratory $(\mathrm{n}=88,69.8 \%)$, and gastrointestinal $(\mathrm{n}=23,18.3 \%) .78(61.9 \%)$ patients used emergency medical services to get to the ED.

Of all the patients, $32(25.4 \%)$ were categorized as refractory anaphylaxis cases. Baseline characteristics of refractory and non-refractory anaphylaxis patients are presented in Table 3. Patients who were female $(P=0.005)$, had DM $(P=0.045)$, presented with cardiovascular symptoms $(P=0.015)$ and were with lower initial MAPs $(P=0.034)$ were more likely to suffer from refractory anaphylaxis. Laboratory findings of the two groups are presented in Table 4 . The median value of NLR was 0.96 (IQR 0.66-1.74). NLR was significantly lower in patients with refractory anaphylaxis, 0.70 (IQR 0.45-1.11), than in those with non-refractory anaphylaxis, 1.11 (IQR 0.721.92), with $P<0.001$ (Fig. 2).

In univariate logistic regression analysis for refractory anaphylaxis, NLR, female sex, DM, malignancy, cardiovascular symptoms, MAP, and body temperature were selected as variables to be included in the multivariate logistic regression model (Table 5). According to the multivariate logistic regression analysis, NLR was independently and inversely associated with the occurrence of refractory anaphylaxis (adjusted odds ratio (OR) $0.33,95 \%$ confidence interval $(\mathrm{CI}) 0.13-0.81, P=$ 0.016).

The ROC curve analysis of NLR to predict refractory anaphylaxis is displayed in Fig. 3. NLR was able to predict refractory anaphylaxis (AUC $=0.717,95 \%$ CI $0.618-0.816, P$ $<0.001)$. The optimal cut-off value of NLR was $<0.68$ using the Youden index, with $50.0 \%$ sensitivity (95\% CI 31.9-68.1) and $80.9 \%$ specificity (95\% CI 71.4-88.2).

\section{Discussion}

To our knowledge, this is the first study on the association between NLR and anaphylaxis refractory to epinephrine treatment. We found that in contrast to previous studies on different allergic diseases, lower NLRs, not higher NLRs, were associated with refractory anaphylaxis refractory to treatment, even after adjusting for possible covariates. Our findings are valuable because refractory anaphylaxis may require additional treatment measures other than epinephrine, such as vasopressors [12], methylene blue [13], or glucagon [14], and early recognition of refractory anaphylaxis may help emergency physicians to make decisions on additional medications 


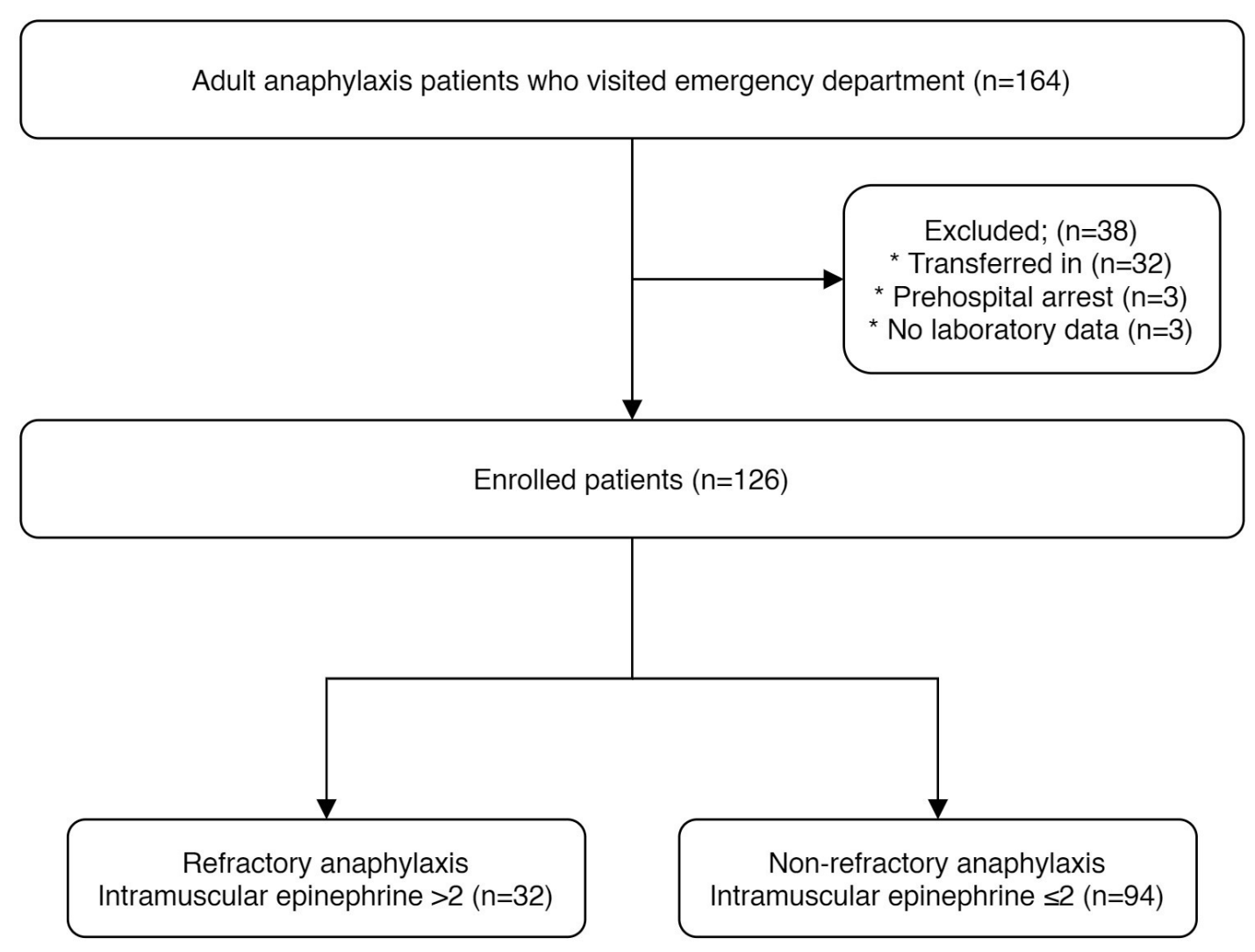

F I G U RE 1. Flow chart of the study design.

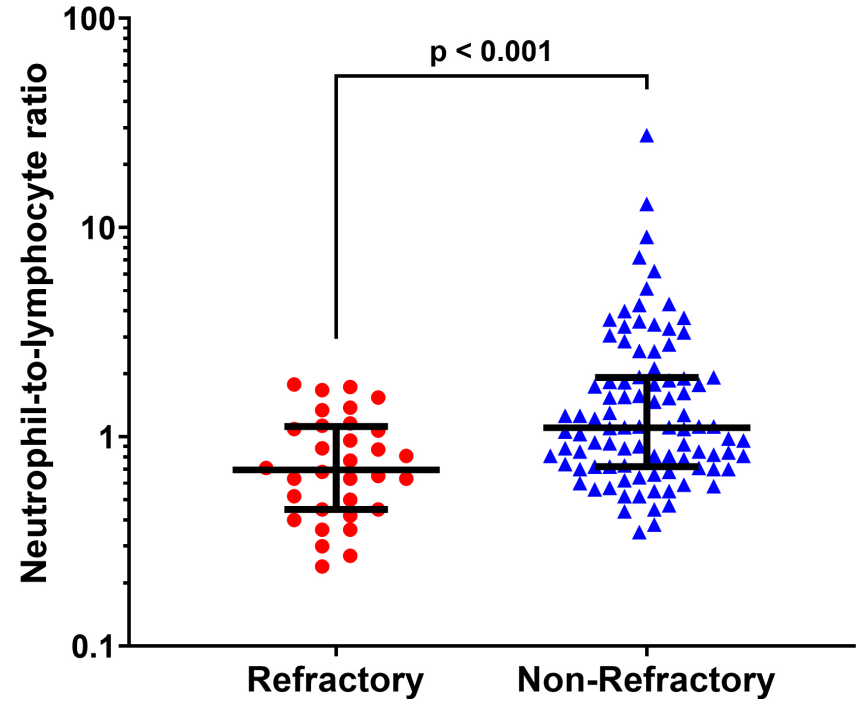

F I G U RE 2. Neutrophil-to-lymphocyte ratios in patients with refractory and non-refractory anaphylaxis, displayed in scatter plots with logarithmic transformation.

and hospital admission.

\subsection{WBC and differential counts in anaphylaxis}

Studies on WBC and differential counts of anaphylaxis patients are still scarce today. A previous study by Tang et al. revealed that both WBC counts and neutrophil percentages were elevated compared with their baseline values in patients

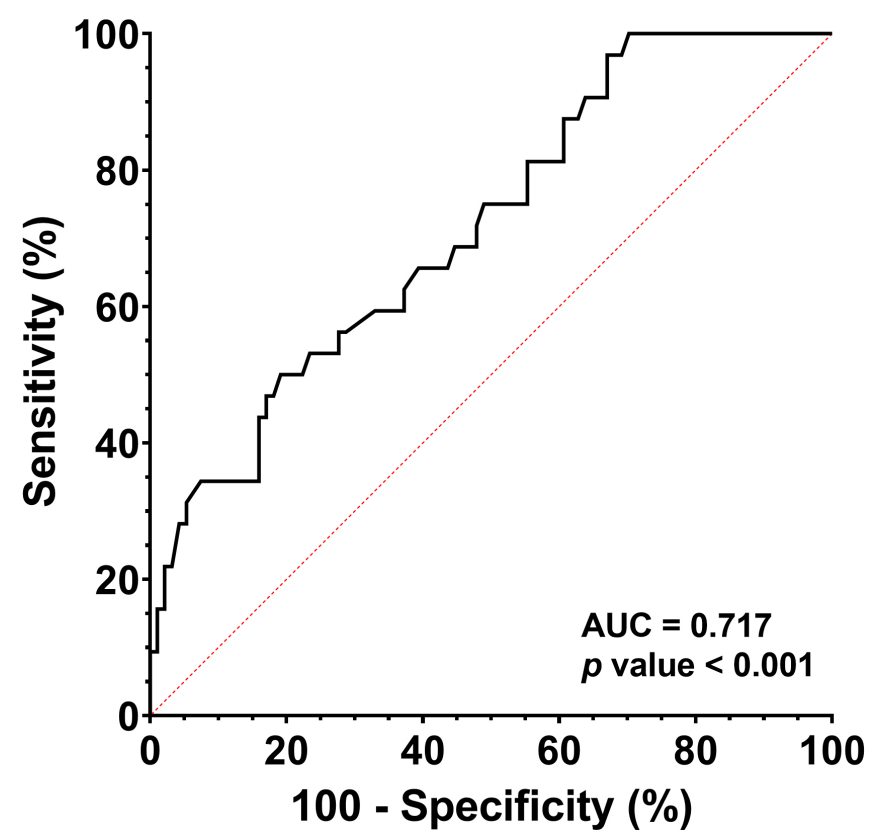

FIGURE 3. Receiver operating characteristics curve analysis of neutrophil-to-lymphocyte ratios to predict refractory anaphylaxis.

with anaphylaxis attacks [15]. However, because all patients included in our study had anaphylaxis attacks, the results may not be directly comparable. A more recent study by Kim et al. compared laboratory results of patients with mild or moderate anaphylaxis reactions with patients with severe anaphylaxis [16]. They were unable to find statistically significant 
T A B L E 3. Baseline characteristics of patients with refractory and non-refractory anaphylaxis.

\begin{tabular}{|c|c|c|c|}
\hline & Refractory & Non-refractory & $P$ value \\
\hline & $\mathrm{n}=32$ & $\mathrm{n}=94$ & \\
\hline Age, years & $56.7 \pm 11.8$ & $53.6 \pm 12.9$ & 0.232 \\
\hline Sex, male & $11(34.4)$ & $59(62.8)$ & 0.005 \\
\hline \multicolumn{4}{|l|}{ Previous medical history } \\
\hline Hypertension & $10(32.3)$ & $25(26.6)$ & 0.613 \\
\hline Diabetes mellitus & $7(22.6)$ & $8(8.5)$ & 0.045 \\
\hline Cardiovascular diseases & $3(9.4)$ & $5(5.3)$ & 0.418 \\
\hline Pulmonary diseases & $2(6.3)$ & $3(3.2)$ & 0.600 \\
\hline Malignancy & $3(9.4)$ & $2(2.1)$ & 0.103 \\
\hline Known allergies to elicitors & $13(40.6)$ & $30(31.9)$ & 0.371 \\
\hline Use of emergency medical services & $18(56.2)$ & $60(63.8)$ & 0.448 \\
\hline Elicitors & & & 0.032 \\
\hline Foods & $3(9.4)$ & $21(22.3)$ & \\
\hline Drugs & $14(43.7)$ & $38(40.4)$ & \\
\hline Contrast media & $6(18.8)$ & $3(3.2)$ & \\
\hline Insect venom & $5(15.6)$ & $24(25.5)$ & \\
\hline Others & $1(3.1)$ & $1(1.1)$ & \\
\hline Idiopathic & $3(9.4)$ & $7(7.4)$ & \\
\hline \multicolumn{4}{|l|}{ Symptoms } \\
\hline Skin \& mucous tissue & $25(78.1)$ & $81(86.2)$ & 0.284 \\
\hline Cardiovascular & $31(96.9)$ & $74(78.7)$ & 0.015 \\
\hline Respiratory & $22(68.8)$ & $66(70.2)$ & 0.877 \\
\hline Gastrointestinal & $5(15.6)$ & $18(19.1)$ & 0.657 \\
\hline \multicolumn{4}{|l|}{ Initial vital signs } \\
\hline Mean arterial pressure, $\mathrm{mmHg}$ & $59(48-76)$ & $69(56-86)$ & 0.034 \\
\hline Heart rate, bpm & $92(83-106)$ & $92(77-102)$ & 0.423 \\
\hline Body temperature, ${ }^{\circ} \mathrm{C}$ & $36.1(36.0-36.5)$ & $36.4(36.0-36.8)$ & 0.118 \\
\hline \multicolumn{4}{|l|}{ Treatment } \\
\hline Number of intramuscular epinephrine injections & $2(2-3)$ & $1(1-2)$ & $<0.001$ \\
\hline Intravenous epinephrine doses, $\mathrm{mg}$ & $0.10(0.00-0.44)$ & $0(0.00-0.00)$ & $<\mathbf{0 . 0 0 1}$ \\
\hline Vasopressor use other than epinephrine & $16(50.0)$ & $0(0)$ & $<0.001$ \\
\hline $\mathrm{H}_{1}$ blocker & $31(96.9)$ & $94(100)$ & 0.254 \\
\hline $\mathrm{H}_{2}$ blocker & $15(46.9)$ & $48(51.1)$ & 0.684 \\
\hline Corticosteroid & $30(93.8)$ & $93(98.9)$ & 0.158 \\
\hline \multicolumn{4}{|l|}{ Disposition } \\
\hline Hospital admission & $27(84.4)$ & $31(33.0)$ & $<0.001$ \\
\hline Hospital length of stay, days & $2(1-2)$ & $1(1-1)$ & $<\mathbf{0 . 0 0 1}$ \\
\hline
\end{tabular}

Data are expressed as numbers (\%) for categorical variables, means \pm standard deviation for normally distributed continuous variables, and medians (interquartile range) for non-normally distributed continuous variables. A P value $<0.05$ is considered statistically significant and is bolded.

differences in WBC counts between groups. However, their study did not focus on the differences in neutrophil counts, lymphocyte counts, or NLRs between groups. Furthermore, their categorization of the study patients was different from ours. Therefore, the findings in our study are relatively new.
Low NLRs indicate that either neutrophil counts are decreased or lymphocyte counts are increased in the blood sample. In our study, patients with refractory anaphylaxis showed lower neutrophil percentages, lower absolute segmented neutrophil counts, and higher lymphocyte percentages than those 
TA B L E 4. Laboratory findings of patients with refractory and non-refractory anaphylaxis.

\begin{tabular}{|lccc|} 
& Refractory & Non-refractory & $P$ value \\
\hline Hemoglobin, g/dL & $\mathrm{n}=32$ & $\mathrm{n}=94$ & \\
\hline Platelet, $10^{9} / \mathrm{L}$ & $14.4 \pm 1.4$ & $15.1 \pm 2.1$ & $\mathbf{0 . 0 4 1}$ \\
\hline White blood cell count, $10^{9} / \mathrm{L}$ & $260 \pm 46$ & $262 \pm 67$ & 0.891 \\
\hline Percentage of segmented neutrophils, $\%$ & $39.19 \pm 2.89$ & $10.02 \pm 3.57$ & $\mathbf{0 . 0 1 0}$ \\
\hline Percentage of lymphocytes, \% & $55.8(43.4-66.7)$ & $44.7(30.5-54.0)$ & $<\mathbf{0 . 0 0 1}$ \\
\hline Percentage of monocytes, \% & $3.9(3.0-5.7)$ & $5.4(4.1-6.6)$ & $\mathbf{0 . 0 1 2}$ \\
\hline Percentage of eosinophils, \% & $0.7(0.5-1.4)$ & $1.0(0.5-1.8)$ & 0.156 \\
\hline Percentage of basophils, \% & $0.2(0.1-0.2)$ & $0.1(0.0-0.2)$ & 0.420 \\
\hline Segmented neutrophils, $10^{9} / \mathrm{L}$ & $2.77(2.25-3.94)$ & $4.54(3.28-6.71)$ & $<\mathbf{0 . 0 0 1}$ \\
\hline Lymphocytes, $10^{9} / \mathrm{L}$ & $4.03(2.77-5.67)$ & $3.62(2.68-5.09)$ & 0.193 \\
\hline Monocytes, $10^{9} / \mathrm{L}$ & $0.33(0.25-0.41)$ & $0.49(0.33-0.73)$ & $<\mathbf{0 . 0 0 1}$ \\
\hline Eosinophils, $10^{9} / \mathrm{L}$ & $0.06(0.03-0.13)$ & $0.09(0.04-0.15)$ & 0.062 \\
\hline Basophils, $10^{9} / \mathrm{L}$ & $0.01(0.01-0.02)$ & $0.01(0.00-0.02)$ & 0.993 \\
\hline Glucose, $\mathrm{mg} / \mathrm{dL}$ & $157(105-186)$ & $158(122-194)$ & 0.342 \\
\hline Blood urea nitrogen, mg/dL & $15.6 \pm 3.9$ & $16.4 \pm 4.8$ & 0.384 \\
\hline Creatinine, mg/dL & $1.00(0.89-1.22)$ & $1.13(0.96-1.35)$ & $\mathbf{0 . 0 4 9}$ \\
\hline Sodium, mmol/L & $142(140-143)$ & $142(140-143)$ & 0.566 \\
\hline Potassium, mmol/L & $3.7 \pm 0.5$ & $3.8 \pm 0.4$ & 0.411 \\
\hline Chloride, mmol/L & $105 \pm 3$ & $104 \pm 3$ & 0.605 \\
\hline C-reactive protein, mg/L & $0.9(0.4-1.7)$ & $1.0(0.5-2.3)$ & 0.390 \\
\hline Neutrophil-to-lymphocyte ratio & $0.70(0.45-1.11)$ & $1.11(0.72-1.92)$ & $<\mathbf{0 . 0 0 1}$ \\
\hline
\end{tabular}

Data are expressed as means \pm standard deviation for normally distributed continuous variables, and medians (interquartile range) for non-normally distributed continuous variables. A P value $<0.05$ is considered statistically significant and is bolded.

with non-refractory anaphylaxis, while there were no statistical differences in absolute lymphocyte counts. This means that there was a decrease in absolute segmented neutrophil counts in patients with refractory anaphylaxis. Similar findings were described previously by Yanagawa et al. [17]. Their study had shown that patients with anaphylactic shock, compared with patients with hemorrhagic, cardiogenic, or septic shock, had relatively decreased neutrophil and increased lymphocyte percentages. Although the study population was different from ours, it partly corroborates our findings in that more severe anaphylaxis patients show decreased neutrophil and increased lymphocyte percentages in $\mathrm{WBC}$ and differential tests.

\subsection{Neutropenia in refractory anaphylaxis}

The mechanism behind neutropenia in refractory anaphylaxis is difficult to speculate. Traditionally, it is believed that anaphylactic reactions are induced by mast cells or basophils [18]. Both IgE antibodies and circulating immune complexes (IC) are known to trigger mast cells and basophils to release mediators, which cause smooth muscle spasm, vasodilation, myocardial depression, and laryngeal edema [2, 19]. However, previous animal studies have shown that neutrophils also play a significant role in anaphylaxis [20]. Neutrophils are considered to be immediate effectors of anaphylactic reactions by directly encountering IC, particularly those formed of IgGs $[2,21]$. Neutrophils activated by ICs are known to release platelet-activating factors, leukotrienes, and histamines, which are all potent mediators of severe allergic reactions [22]. This may explain the elevation of neutrophil counts in patients with anaphylaxis attacks because neutrophil activation will mobilize neutrophils to enter the bloodstream [23]. Also, it is surmised that patients with more severe anaphylactic reactions may have a greater number of neutrophils, which is contrary to the results of our study [24].

One possible explanation is that refractory anaphylaxis may utilize a different immune pathway from a typical anaphylaxis. It is presumed that in contrast to non-refractory anaphylaxis, nitric oxide (NO) may play a critical role in refractory anaphylaxis [19]. Platelet-activating factors, leukotrienes, and histamines may enhance NO production by activating endothelial cell NO synthases, which activate guanylate cyclase to synthesize cyclic guanylate monophosphate (cGMP) from guanosine triphosphate [25]. Overproduction of NO and cGMP contributes to vasodilation and distributive shock [13]. Anaphylactic shock induced by this NO-cGMP pathway may not rapidly respond to standard intramuscular epinephrine treatment [26]. A previous in vitro study had shown that NO 
T A B L E 5. Univariate and multivariate logistic regression analysis for predicting refractory anaphylaxis.

\begin{tabular}{|c|c|c|c|c|c|c|}
\hline & \multicolumn{3}{|c|}{ Crude } & \multicolumn{3}{|c|}{ Adjusted } \\
\hline & OR & $95 \% \mathrm{CI}$ & $P$ value & OR & $95 \% \mathrm{CI}$ & $P$ value \\
\hline Neutrophil-to-lymphocyte ratio & 0.28 & $0.12-0.68$ & 0.005 & 0.33 & $0.13-0.81$ & 0.016 \\
\hline Age, years & 1.02 & $0.99-1.06$ & 0.231 & & & \\
\hline Female sex & 3.22 & $1.39-7.46$ & 0.006 & 2.77 & $1.07-7.17$ & 0.035 \\
\hline \multicolumn{7}{|l|}{ Previous medical history } \\
\hline Hypertension & 1.25 & $0.52-3.01$ & 0.612 & & & \\
\hline Diabetes mellitus & 3.01 & $0.99-9.11$ & 0.051 & 3.75 & $0.95-14.83$ & 0.059 \\
\hline Cardiovascular diseases & 1.84 & $0.41-8.18$ & 0.422 & & & \\
\hline Pulmonary diseases & 2.02 & $0.32-12.68$ & 0.452 & & & \\
\hline Malignancy & 4.76 & $0.76-29.88$ & 0.096 & 3.08 & $0.33-28.43$ & 0.321 \\
\hline Known allergies to elicitors & 1.46 & $0.64-3.34$ & 0.371 & & & \\
\hline Use of emergency medical services & 0.73 & $0.32-1.65$ & 0.447 & & & \\
\hline \multicolumn{7}{|l|}{ Symptoms } \\
\hline Skin \& mucous tissue & 0.57 & $0.21-1.59$ & 0.286 & & & \\
\hline Cardiovascular & 8.38 & $1.08-65.19$ & 0.042 & 4.41 & $0.43-44.90$ & 0.210 \\
\hline Respiratory & 0.93 & $0.39-2.22$ & 0.876 & & & \\
\hline Gastrointestinal & 0.78 & $0.26-2.31$ & 0.656 & & & \\
\hline \multicolumn{7}{|l|}{ Initial vital signs } \\
\hline Mean arterial pressure, $\mathrm{mmHg}$ & 0.98 & $0.96-1.00$ & 0.057 & 1.00 & $0.97-1.02$ & 0.831 \\
\hline Heart rate, bpm & 1.01 & $0.99-1.03$ & 0.510 & & & \\
\hline Body temperature, ${ }^{\circ} \mathrm{C}$ & 0.47 & $0.19-1.15$ & 0.099 & 1.05 & $0.35-3.13$ & 0.925 \\
\hline \multicolumn{7}{|l|}{ Treatment } \\
\hline $\mathrm{H}_{2}$ blocker & 0.85 & $0.38-1.89$ & 0.682 & & & \\
\hline Corticosteroids & 0.16 & $0.01-1.84$ & 0.142 & & & \\
\hline
\end{tabular}

Variables with $P$ value $<0.1$ are included in the multivariate logistic regression model. A P value $<$ 0.05 is considered statistically significant and is bolded. Abbreviations: OR, odds ratio; $C I$, confidence interval.

mediates the apoptosis of neutrophils by enhanced reactive oxygen species production and caspase- 8 activation [27]. It is possible that overproduced NO may accelerate neutrophil apoptosis, resulting in neutropenia. However, the relationship between the NO-cGMP pathway and lower absolute segmented neutrophil counts is still not fully understood, and future studies are warranted.

\subsection{Incidence of refractory anaphylaxis}

The incidence of refractory anaphylaxis in our study (25.4\%) was much more frequent than described in the literature. A previous study by Korenblat et al. reported that 17 out of 105 patients $(16.2 \%)$ required three or more intramuscular epinephrine injections [28]. A more recent analysis of the European Anaphylaxis Registry by Francuzik et al. reported that only less than $1 \%$ of severe anaphylaxis cases were refractory to epinephrine treatment [6]. The variation in disease prevalence may be because of epidemiologic factors. During the study period in South Korea, epinephrine autoinjectors were only available at the Korea Orphan and Essential Drug Center with a prescription, and intramuscular epinephrine injection by emergency medical technicians was prohibited by law. Thus, a vast majority of anaphylaxis patients received intramuscular epinephrine only after arriving at the hospital. Delays in epinephrine treatment is associated with more severe, even fatal anaphylactic reactions [29]. This may have caused more frequent refractory anaphylaxis events than observed in previous studies. Also, the treatment protocol of our emergency center may be more aggressive than the standard care given at other centers; 110 out of 126 patients $(87.3 \%)$ received intramuscular epinephrine injections, which is more frequent than previously described, and it may have affected the ratio [30].

\subsection{Use of point-of-care testing devices}

Since anaphylaxis is a medical emergency requiring clinical diagnosis and immediate management, laboratory tests such as $\mathrm{CBCs}$ may not be performed rapidly enough to influence emergency physicians' treatment decisions. One potential solution to the problem may be the utilization of point-of-care testing (POCT) devices. There are several commercial POCT devices to accurately measure WBC and differential counts 
within minutes [31, 32]. This approach has been previously studied in severe asthma, and it has shown promising results [33]. These POCT devices to obtain NLRs may be used as an alternative to traditional $\mathrm{CBC}$ teststest, but its use in anaphylaxis may need to be validated.

\subsection{Limitations}

Our study had several limitations. This was a single-center, retrospective study with a small sample size, so it was exposed to potential confounders and selection bias. A multicenter prospective study is required to confirm our findings. Refractory anaphylaxis is still not clearly defined, and our definition is not universally accepted. A consensus for a definition of refractory anaphylaxis may be needed. Most patients did not undergo skin or provocation tests to confirm the elicitors of their anaphylactic reactions. The causes of anaphylaxis may not be accurate because we relied solely on patient history. The blood samples used for analysis were usually taken as soon as the patient arrived at our hospital, but the exact timing of blood sampling and intramuscular epinephrine injection were not clarified, and epinephrine injections may have influenced $\mathrm{CBC}$ and differential test results. Additionally, some patients may have taken medications before arriving at our emergency department that may have affected results, including allergy medications and chemotherapeutic agents, and that were not fully addressed in the medical records.

\section{Conclusions}

NLR is independently and inversely associated with the occurrence of refractory anaphylaxis among patients with anaphylaxis. The optimal cut-off value to predict refractory anaphylaxis was NLR $<0.68$. NLR may be used as an easy and inexpensive test to predict refractory anaphylaxis. However, future studies are warranted to confirm these findings.

\section{AUTHOR CONTRIBUTIONS}

Kiwook Kim and Kyoung Ho Choi designed the study. Joo Suk Oh, Doo Hyo Lee, Hyun Ho Jeong, Young Min Oh, and Se Min Choi collected the data. Jung Taek Park analyzed the data. Kiwook Kim and Kyoung Ho Choi analyzed the results and drafted the manuscript.

\section{ETHICS APPROVAL AND CONSENT TO PARTICIPATE}

Our study was approved by the Institutional Review Board of Uijeongbu St. Mary's Hospital on March 25, 2020 (number UC20RASI0037).

\section{ACKNOWLEDGMENT}

Thank numerous individuals participated in this study.

\section{FUNDING}

This research received no specific grant from any funding agency in the public, commercial, or not-for-profit sectors.

\section{CONFLICT OF INTEREST}

The authors declare that there is no conflict of interest regarding the publication of this article.

\section{DATA AVAILABILITY}

The data used to support the findings of this study are available from the corresponding author upon request.

\section{REFERENCES}

[1] Simons FER, Ardusso LRF, Bilò MB, Cardona V, Ebisawa M, El-Gamal YM, et al. International consensus on (ICON) anaphylaxis. World Allergy Organization Journal. 2014; 7 : 9.

[2] Reber LL, Hernandez JD, Galli SJ. The pathophysiology of anaphylaxis. The Journal of Allergy and Clinical Immunology. 2017; 140: 335-348.

[3] Kemp SF, Lockey RF, Simons FER. Epinephrine: the drug of choice for anaphylaxis. a statement of the World Allergy Organization. Allergy. 2008; 63: 1061-1070.

[4] Ma L, Danoff TM, Borish L. Case fatality and population mortality associated with anaphylaxis in the United States. The Journal of Allergy and Clinical Immunology. 2014; 133: 1075-1083.

[5] Francuzik W, Dölle S, Worm M. Risk factors and treatment of refractory anaphylaxis - a review of case reports. Expert Review of Clinical Immunology. 2018; 14: 307-314.

[6] Francuzik W, Dölle-Bierke S, Knop M, Scherer Hofmeier K, CichockaJarosz E, García BE, et al. Refractory anaphylaxis: data from the european anaphylaxis registry. Frontiers in Immunology. 2019; 10: 2482.

[7] Dogru M, Evcimik MF, Cirik AA. Is neutrophil-lymphocyte ratio associated with the severity of allergic rhinitis in children? European Archives of Oto-Rhino-Laryngology. 2016; 273: 3175-3178.

[8] Jiang Y, Ma W. Assessment of neutrophil-to-lymphocyte ratio and platelet-to-lymphocyte ratio in atopic dermatitis patients. Medical Science Monitor : International Medical Journal of Experimental and Clinical Research. 2017; 23: 1340-1346.

[9] Mochimaru T, Ueda S, Suzuki Y, Asano K, Fukunaga K. Neutrophil-tolymphocyte ratio as a novel independent predictor of severe exacerbation in patients with asthma. Annals of Allergy, Asthma \& Immunology. 2019; 122: 337-339.e331.

[10] Sampson HA, Muñoz-Furlong A, Campbell RL, Adkinson NF, Bock SA, Branum A, et al. Second symposium on the definition and management of anaphylaxis: summary report-second National Institute of Allergy and Infectious Disease/Food Allergy and Anaphylaxis Network symposium. Annals of Emergency Medicine. 2006; 47: 373-380.

[11] Brown SGA. Clinical features and severity grading of anaphylaxis. The Journal of Allergy and Clinical Immunology. 2004; 114: 371-376.

[12] Alqurashi W, Alnaji F, Menon K. Refractory anaphylaxis: further considerations for emergency care providers. Annals of Allergy, Asthma \& Immunology. 2016; 116: 265-266.

[13] Jang DH, Nelson LS, Hoffman RS. Methylene blue for distributive shock: a potential new use of an old antidote. Journal of Medical Toxicology. 2013; 9: 242-249.

[14] Rukma P. Glucagon for refractory anaphylaxis. American Journal of Therapeutics. 2019; 26: e755-e756.

[15] Tang R, Xu H, Cao J, Chen S, Sun J, Hu H, et al. Clinical characteristics of inpatients with anaphylaxis in China. BioMed Research International. 2015; 2015: 429534.

[16] Kim S, Kim M, Cho Y. Different clinical features of anaphylaxis according to cause and risk factors for severe reactions. Allergology International. 2018; 67: 96-102.

[17] Yanagawa Y, Sakamoto T, Okada Y. Lymphocytosis without anemia in 
a patient presenting with anaphylactic shock. The American Journal of Emergency Medicine. 2005; 23: 763-766.

[18] Kemp SF, Lockey RF. Anaphylaxis: a review of causes and mechanisms. The Journal of Allergy and Clinical Immunology. 2002; 110: 341-348.

[19] LoVerde D, Iweala OI, Eginli A, Krishnaswamy G. Anaphylaxis. Chest. 2018; 153: 528-543.

[20] Jönsson F, Mancardi DA, Kita Y, Karasuyama H, Iannascoli B, Van Rooijen N, et al. Mouse and human neutrophils induce anaphylaxis. The Journal of Clinical Investigation. 2011; 121: 1484-1496.

[21] Jönsson F, Mancardi DA, Albanesi M, Bruhns P. Neutrophils in local and systemic antibody-dependent inflammatory and anaphylactic reactions. Journal of Leukocyte Biology. 2013; 94: 643-656.

[22] Feuerstein G, Hallenbeck JM. Prostaglandins, leukotrienes, and plateletactivating factor in shock. Annual Review of Pharmacology and Toxicology. 1987; 27: 301-313.

[23] Rosales C, Lowell CA, Schnoor M, Uribe-Querol E. Neutrophils: their role in innate and adaptive immunity 2017. Journal of Immunology Research. 2017; 2017: 9748345.

[24] Francis A, Bosio E, Stone SF, Fatovich DM, Arendts G, Nagree Y, et al. Neutrophil activation during acute human anaphylaxis: analysis of MPO and sCD62L. Clinical and Experimental Allergy. 2017; 47: 361-370.

[25] Mitsuhata H, Shimizu R, Yokoyama MM. Role of nitric oxide in anaphylactic shock. Journal of Clinical Immunology. 1995; 15: 277-283.

[26] Evora PRB, Simon MR. Role of nitric oxide production in anaphylaxis and its relevance for the treatment of anaphylactic hypotension with methylene blue. Annals of Allergy, Asthma \& Immunology. 2007; 99: 306-313.

[27] Dubey M, Nagarkoti S, Awasthi D, Singh AK, Chandra T, Kumaravelu $\mathrm{J}$, et al. Nitric oxide-mediated apoptosis of neutrophils through caspase-
8 and caspase-3-dependent mechanism. Cell Death \& Disease. 2016; 7 : e2348.

[28] Korenblat P, Lundie MJ, Dankner RE, Day JH. A retrospective study of epinephrine administration for anaphylaxis: how many doses are needed? Allergy and Asthma Proceedings. 1999; 20: 383-386.

[29] Bernstein DI, Wanner M, Borish L, Liss GM. Twelve-year survey of fatal reactions to allergen injections and skin testing: 1990-2001. The Journal of Allergy and Clinical Immunology. 2004; 113: 1129-1136.

[30] Sclar DA, Lieberman PL. Anaphylaxis: underdiagnosed, underreported, and undertreated. The American Journal of Medicine. 2014; 127: S1-S5.

[31] Karawajczyk M, Haile S, Grabski M, Larsson A. The HemoCue WBC DIFF system could be used for leucocyte and neutrophil counts but not for full differential counts. Acta Paediatrica. 2017; 106: 974-978.

[32] Majors CE, Pawlowski ME, Burke DC, Tkaczyk TS, Rieber A, RichardsKortum R. Clinical training and validation of the LeukoScope: a low-cost, point-of-care device to perform white blood cell and neutrophil counts. RSC Advances. 2019; 9: 27324-27333.

[33] Heffler E, Terranova G, Chessari C, Frazzetto V, Crimi C, Fichera S, et al. Point-of-care blood eosinophil count in a severe asthma clinic setting. Annals of Allergy, Asthma \& Immunology. 2017; 119: 16-20.

How to cite this article: Kiwook Kim, Kyoung Ho Choi, Jung Taek Park, Joo Suk Oh, Doo Hyo Lee, Hyun Ho Jeong, et al. The association between neutrophil-to-lymphocyte ratio and anaphylaxis refractory to epinephrine treatment. Signa Vitae. 2021;17(3):158-166. doi:10.22514/sv.2021.034. 